This is the peer reviewed version of the following article: V. Valderrey, A. Bonasera, S. Fredrich, S. Hecht, Angew. Chem. Int. Ed. 2017, 56, 1914, which has been published in final form at http://onlinelibrary.wiley.com/doi/10.1002/anie.201609989/abstract. This article may be used for noncommercial purposes in accordance with Wiley Terms and Conditions for Self-Archiving. Supporting Information is available free of charge following the previous link.

\title{
Light-Activated Sensitive Probes for Amine Detection
}

\author{
Virginia Valderrey ${ }^{+}$, Aurelio Bonasera ${ }^{+}$, Sebastian Fredrich and Stefan Hecht*
}

\begin{abstract}
Our new, simple, and accurate colorimetric method is based on diarylethenes (DAEs) for the rapid detection of a wide range of primary and secondary amines. The probes consist of aldehyde- or ketone-substituted diarylethenes, which undergo an amine-induced decoloration reaction, selectively to give the ring-closed isomer. Thus, these probes can be activated at the desired moment by light irradiation, with a sensitivity that allows the detection of amines at concentrations as low as $10^{-6} \mathrm{M}$ in solution. In addition, the practical immobilization of DAEs on paper makes it possible to detect biogenic amines, such as cadaverine, in the gas phase above a threshold of 12 ppbv within 30 seconds.
\end{abstract}

Amines are ubiquitous natural constituents of all living beings (i.e. amino acids, neurotransmitters, etc.) and beyond certain concentrations some of them can be indicative of industrial effluvia or food spoilage. ${ }^{[1]}$ Various biogenic amines, such as cadaverine, histamine, tyramine, putrescine, spermidine, spermine, and ethanolamine are products of thermal or enzymatic decarboxylation of amino acids by bacteria. ${ }^{[2]}$ Consequently, the accumulation of these amines can serve as an indicator of food quality or hygiene. ${ }^{[3]}$ The detection and discrimination of compounds related to a given environmental hazard continues to challenge the scientific community and therefore, amine sensing has been widely investigated with several detection protocols described in the literature. Of these, chromatographic techniques coupled with mass spectrometry are preferentially employed, because they provide good detection limits towards amines. ${ }^{[4]}$ However, these methods are limited by extended analysis times as well as expensive and large instrumentation. Antibodies and enzymes have been employed and have also provided good sensitivities; ${ }^{[5]}$ nevertheless, the main drawback of these approaches is the use of materials that are sensitive to chemicals and temperature. In addition, antibodies have to be raised, representing a limiting factor in the availability of the active materials. Several colorimetric sensors based on simple chromophores, which recognize amines by noncovalent interactions, have been designed in order to overcome those drawbacks. Different concepts for amine sensing, such as Suslick's colorimetric arrays, ${ }^{[6]}$ Lavigne's polythiophene carboxylic acids, ${ }^{[7]}$ Anslyn's receptors, ${ }^{[8]}$ 
Severin's coumarin probe, ${ }^{[9]}$ Miljanić's cruciform, ${ }^{[10]}$ and Kaneda's cyclodextrin dye,${ }^{[11]}$ have been developed. A colorimetric sensor for amines that combines enzymes and chromophores has also been described. ${ }^{[12]}$ More recently, photochromic materials have been employed as sensing units for the detection of different analytes, such as amino acids, cyanide anions, thiols, and organophosphorus compounds. ${ }^{[13]}$ In these examples, the reactivity of the chromophore can be triggered by means of irradiation with UV or visible light. This approach is more suitable for practical applications, since the sensor can be stored in its dormant form until the activation of the molecule shortly before the use. Moreover, the pre-exposure of the inactive sensor to an amine-containing environment will not affect the performance of the active form, thus preventing overestimation of the analyte detection. Herein, we describe a methodology for the efficient recognition and quantification of amines by specific diarylethenes (DAEs), which can be activated at the desired point in time and at defined areas of a surface using light as a remote control, without influencing the local concentration.

DAEs typically undergo $6 \pi$-electrocyclization and cycloreversion reactions upon irradiation with UV and visible light, respectively. ${ }^{[14]}$ This photochemical reaction is accompanied by visible color changes: the open isomer, absorbing in the near-UV region, is converted into the closed one, with distinct red-shifted absorption, in a reversible manner. In addition, the electronic changes originating from the photoisomerization make it possible to control the reactivity of properly substituted DAEs, for example aldehyde-substituted DAEs in condensation reactions with amines. ${ }^{[15]}$ Inspired by these examples, we envisaged that DAEs containing a carbonyl group at the end of the hexatriene system should experience an even more dramatic change in the reactivity towards amines. We therefore synthesized a series of photoswitchable DAEs, functionalized at the reactive position of one of the two heteroaryl fragments with a formyl (10-3o) or an acetyl (40) group (Scheme 1). Ring-closure of open DAEs (1o-4o) with UV light converts the less reactive aromatic carbonyl group into a more reactive aliphatic one. ${ }^{[16]}$

UV/Vis absorption spectroscopy of a $4 \times 10^{-5} \mathrm{~m}$ solution of 10 in acetonitrile indicates that the open isomer 10 undergoes an electrocyclization reaction upon irradiation with $365 \mathrm{~nm}$ light (see Figure S10 in the Supporting Information). This is evidenced by the formation of a band in the visible region $\left(\lambda_{\max }=564 \mathrm{~nm}\right)$, which implies a change in the color of the solution from colorless to violet. Ultraperformance liquid chromatography (UPLC) analysis of the irradiated solution shows that in the photostationary state (PSS) approximately $80 \%$ of $\mathbf{1 o}$ has been converted to the closed isomer 1c. Irradiation of the solution of $\mathbf{1}$ in the PSS with $500 \mathrm{~nm}$ light recovers the pure open isomer 10. This process can be repeated for several cycles (see Figure S20 in the Supporting Information and Figures S15-S19 for thermal stability). The reactivity of the two DAE isomers towards amines was initially investigated by ${ }^{1} \mathrm{H}$ NMR spectroscopy (Figure 1, bottom). Irradiation of a colorless millimolar solution of $\mathbf{1 0}$ with light at $350 \mathrm{~nm}$ for $30 \mathrm{~min}$ produces a deep violet solution with a ${ }^{1} \mathrm{H}$ NMR spectrum containing diagnostic signals for the open and closed isomers in a ratio close to that of the PSS as determined by UPLC for the $4 \times 10^{-5} \mathrm{M}$ solution. The addition of 1.0 equiv of benzylamine (BA) to the irradiated DAE mixture immediately causes decoloration of the solution. 
After $30 \mathrm{~min}$, the ${ }^{1} \mathrm{H}$ NMR signals of 10 remain unaffected, indicating that the open DAE isomer is completely unreactive towards the amine. In contrast, in the case of the closed isomer 1c the proton signals change. The aldehyde signal of 1c disappears, suggesting that the nucleophilic addition of the amine to the aldehyde group took place selectively only in the closed isomer. Similar results are obtained by UV/Vis absorption spectroscopy. The addition of BA to a solution of 1o, irradiated at $365 \mathrm{~nm}$ to achieve the PSS, results in a gradual decay of the band centered at $\lambda_{\max }=564 \mathrm{~nm}$ in the visible region of the spectrum and a hypsochromic shift of the band in the UV region is observed, resulting in a yellowish solution (Figure 2a). Once no further changes of the absorption spectra were observed, the sample was irradiated again with 365 $\mathrm{nm}$ light but no further spectral changes occurred. In view of these results, we conclude that 1c reacts selectively with BA in the presence of 10 and in an irreversible manner to generate reaction product $1 \mathrm{r}$, which is photoinactive. In order to elucidate the structure of $\mathbf{1 r}$, we isolated the closed isomer 1c, which was subsequently treated with 1.0 equiv of BA (further details are given in the Supporting Information). X-ray diffraction analysis of pure $1 \mathrm{r}$ revealed that the DAE core rearranges into a benzothiophene scaffold, ${ }^{[17]}$ causing the decomposition of the thiophene ring opposite to the aldehyde, generating a photochemically unreactive compound (Figure 2b).

The presence of $N$-benzylformamide as a by-product in the ${ }^{1} \mathrm{H}$ NMR experiments provides further insight into a reasonable mechanism for the observed rearrangement reaction. Initially, the nucleophilic attack of the amine at the highly reactive, aliphatic aldehyde of the closed DAE isomer produces the corresponding hemiaminal intermediate. Subsequently, this intermediate rearranges to the more stable compound 1r with elimination of $N$-benzylformamide (Figure 1, top). To confirm the proposed mechanism for the formation of 1r, we synthesized the imine derivative 1i starting from the open DAE 1o. As expected, 1i can be switched between the open and the closed isomers by alternating UV- and visible-light irradiation in a reversible fashion and without generation of $\mathbf{1 r}$ (Scheme 1, see Figure S35 in the Supporting Information). This is in agreement with the proposed mechanism for the formation of $\mathbf{1 r}$, which requires the intermediacy of the hemiaminal derivative (Figure 1, top). Similar experiments were performed using switches 2-4. UV/Vis absorption and ${ }^{1} \mathrm{H}$ NMR spectra confirmed the transformation of the investigated switches into products that show the same central scaffold as 1r, and differ only in the substitution pattern (for further details see the Supporting Information). The main difference in the amine-induced rearrangements of DAEs $1 \mathbf{c}-\mathbf{4 c}$ is the reaction time, which reflects the reactivity of the carbonyl moiety. As expected, the reactivity follows the order $\mathbf{3 c}>\mathbf{1} \mathbf{c} \approx \mathbf{2} \mathbf{c}>\mathbf{4} \mathbf{c}$ (for the UV/Vis spectra see the Supporting Information). This order can be rationalized considering that the less reactive ketone $\mathbf{4 c}$ forms the hemiaminal intermediate more slowly than the aldehydes, of which $\mathbf{3 c}$ is the fastest as it contains no electron-donating methoxy group. Note that the effect of substitution on the thiophene ring is smaller than that resulting from a slight change in the amine structure, highlighting the degree of chemoselectivity for sensing amines. 
After serendipitously discovering this unique rearrangement reaction of the closed, carbonyl-substituted DAE isomers, we engaged in a systematic spectroscopic investigation in order to 1) quantify the rate constant for the reaction of all four switches with a variety of amines and to 2) determine the lowest concentration range for which an evolution of the spectral bands takes place over a reasonable time frame (up to $2 \mathrm{~h}$ ).

A collection of eight different amines was studied in combination with the synthesized DAE switches (Table 1 and the Supporting Information). Amines were chosen to represent different classes, such as tertiary amines ( $N, N$-dimethylbenzylamine (DMBA)), secondary amines ( $N$-benzylmethylamine (BMA)), bulky primary amines (benzylamine and $(S)-(-)-\alpha$-methylbenzylamine (MBA)), primary linear aliphatic amines (octylamine (OA)), primary diamines (1,3-diaminopropane (DAP) and cadaverine (CAD)), and aliphatic trisamines (spermidine (SPER)). In a typical experiment, a $2 \times 10^{-5} \mathrm{M}$ solution of the DAE was irradiated until it reached its PSS (for the PSS values of the DAEs see Table S2 in the Supporting Information); afterwards a specific amount of amine was added. We monitored the variation of optical density at the maximum of the visible absorption band belonging to the closed isomer. This region was chosen since the absorption of the rearrangement products $\mathbf{1 r}-\mathbf{4 r}$ is minimal and the absorbance change thereby maximized. The rate constants were determined by fitting the UV/Vis spectral changes with a first-order reaction. As expected from the proposed mechanism, tertiary amines are not reactive. Secondary amines react much slower than primary ones as indicated by comparing the rates of BMA with BA. This discrimination is presumably due to the increased steric hindrance of the secondary amines. For similar reasons BA reacts faster than MBA. Purely aliphatic amines are about one order of magnitude more reactive than BA. Finally, aliphatic oligoamines, such as DAP, CAD, and SPER react faster than what would be expected by simply correcting for the number of amino groups present in the corresponding molecules. Clearly, neighboring group effects play an important role in these cases.

When considering these reactivity data, particular attention should be paid to $\mathbf{3 c}$, which displays better photoswitching performance (high PSS), excellent thermal stability (see Figure S18 in the Supporting Information), and also higher reactivity. According to the data obtained for $\mathrm{CAD}$, the rearrangement of $\mathbf{3 c}$ occurs 1.6 times faster than that of $1 \mathrm{c}$ and a limiting concentration of $2.0 \times 10^{-5} \mathrm{M}$ was determined, with a $50 \%$ decrease of the absorption at $580 \mathrm{~nm}$ within $110 \mathrm{~min}$ (see Figure S57 in the Supporting Information).

To optimize the conditions for sensing of the amines we studied the influence of the $\mathrm{pH}$ on the kinetics of the reaction (see Section 6.1. in the Supporting Information). The optimal rate was obtained upon addition of 10 equiv of $p$-toluenesulfonic acid to a solution of $\mathbf{3 c}$ in $\mathrm{CH}_{3} \mathrm{CN}$ followed by the addition of 10 equiv of CAD. This resulted in a 40-fold rate increase as compared to the rate in the absence of the acid catalyst. A larger excess of acid led to decreasing rates. This observation points to the classic scenario of an optimal, slightly acidic $\mathrm{pH}$ window, ${ }^{[19]}$ where a small amount of acid increases the electrophilicity of the carbonyl group yet too large amounts lead to protonation of the amine, thereby reducing its nucleophilicity. 
Once the reaction conditions for amine sensing had been optimized, we focused on applying this methodology by using simple and inexpensive platforms. Initially, we decided to create a colorcode assay, which makes it possible to detect amines with the "naked eye" quickly and easily. Different quantities of amines were added to a 16-well microplate filled with a solution of $\mathbf{3}\left(\mathrm{CH}_{3} \mathrm{CN}, \lambda_{\text {irr }}=365 \mathrm{~nm}\right)$. This resulted in the characteristic color change from deep violet to yellow at different rates. Manipulation of the pictures taken during the colorimetric assay allowed us to create a palette of colors that can be used to estimate the amine concentration in a solution within a short time interval (see Figures S62 and 63 in the Supporting Information).

In order to prove the versatility of our sensing strategy we moved from studies in solution to the preparation of amine-sensitive solid supports. A filter paper was coated with the colorless inactive DAE 3o (Figure 3a). After that, the surface was covered with a photomask and irradiated with $365 \mathrm{~nm}$ light. In this way we could create a color-patterned surface by converting DAE 3o into its active form 3c, in precisely specified areas of the surface (Figure 3b). After the exposure of the sample to vapors of CAD we observed that only the areas containing the light-activated sensor 3c were decolored (Figure 3c). Moreover, the regions that were previously not activated by light can be activated a posteriori (Figure 3d). This makes patterning possible and the sensor can be used multiple times through the sequential activation of specific small areas.

This sequential UV activation nicely demonstrates that the inactive sensing material does not react with the amines until the moment it is activated by the UV light, ensuring that the sensor is preserved until the appropriate moment of amine sensing. Importantly, this methodology allowed us to sense vapors of cadaverine down to a threshold of 12 ppbv (parts per billion in volume) in $30 \mathrm{~s} \mathrm{(see} \mathrm{Figures} \mathrm{S65} \mathrm{and} \mathrm{S66} \mathrm{in}$ the Supporting Information).

In conclusion, we have described a simple method for the detection of amines in solution and in the gas phase. Our colorimetric assay is based on aldehyde-substituted DAE scaffolds, which can be activated by exposure to UV light. Only the on-demand-generated closed isomer can react with amines, leading to a decolorized rearrangement product (and an amide). Kinetic studies involving facile UV/Vis absorption monitoring allowed us to discriminate a wide variety of amines and revealed rapid reaction in the presence of primary aliphatic amines, while secondary amines react more slowly, and tertiary amines do not react at all. In contrast to other methodologies based on multicomponent systems or bimolecular cascade reactions, which require two different sensing molecules, our strategy involves only one. Here, the two sequential events occur simultaneously in the same sensing unit, providing extremely rapid responses. The low reactivity of the open isomer towards amines and the possibility of activating the DAE sensors by irradiation on defined areas of surfaces make them ideal for the development of a new generation of smart yet low-cost sensing devices. Current work in our laboratories is focused on 
1) Enhancing the reactivity of the closed form for the rearrangement by implementing electron-accepting substituents to stabilize the thiolate leaving group (see mechanism Figure 1, top);

2) Implementing fluorescence as a more sensitive readout;

3) Achieving high photoconversion in the PSS upon exposure to sunlight to ease the use in developing countries.

Beyond amine detection, we are aiming to develop this promising new transformation into a light-triggered acyl transfer reaction for bioconjugation.

\section{Acknowledgements}

We thank Dr. Beatrice Cula Braun for the X-ray crystallographic data and Michael Kathan for helpful discussions. Generous support by the European Research Council (ERC via ERC-2012-STG_308117 "Light4Function"), the European Commission (via MSCA-ITN “iSwitch" GA No. 642196), the German Research Foundation (DFG via SFB 658, project B8), and the Alexander von Humboldt Foundation is gratefully acknowledged.

\section{Keywords}

amines $\cdot$ diarylethenes $\cdot$ photochromism $\cdot$ sensing

\section{References}

[1] a) S. Bardocz, Trends. Food. Sci. Tech. 1995, 6, 341-346; b) A. Naila, S. Flint, G. Fletcher, P. Bremer, G. Meerdink, J. Food Sci. 2010, 75, R139-R150.

[2] a) B. ten Brink, C. Damink, H. M. L. J. Joosten, J. H. J. Huis in 't Veld, Int. J. Food. Microbiol. 1990, 11, 73-84; b) A. Halász, Á. Baráth, L. Simon-Sarkadi, W. Holzapfel, Trends. Food. Sci. Tech. 1994, 5, 42-49; c) M. H. S. Santos, Int. J. Food. Microbiol. 1996, 29, 213-231.

[3] a) I. A. Bulushi, S. Poole, H. C. Deeth, G. A. Dykes, Crit. Rev. Food. Sci. 2009, 49, 369-377; b) C. Y. Chong, F. Abu Bakar, A. R. Russly, B. Jamilah, N. A. Mahyudin, Int. Food Res. J. 2011, 18, 867-876.

[4] a) D. C. Johnson, D. Dobberpuhl, R. Roberts, P. Vandeberg, J. Chromatogr. A 1993, 640, 79-96; b) S. Shesnov, L. Bigler, M. Hesse, Eur. J. Mass Spectrom. 2002, 8, 1-16; c) M. L. Latorre-Moratalla, J. BoschFusté, T. Lavizzari, S. Bover-Cid, M. T. Veciana-Nogués, M. C. Vidal-Carou, J. Chromatogr. A 2009, 1216, 7715-7720.

[5] a) J. Lange, C. Wittmann, Anal. and Bioanal. Chem. 2002, 372, 276-283; b) B. Bóka, N. Adányi, D. Virág, M. Sebela, A. Kiss, Electroanal. 2012, 24, 181-186; c) S. Leonardo, M. Campàs, Microchim. Acta 2016, 183, 1881-1890.

[6] a) N. A. Rakow, A. Sen, M. C. Janzen, J. B. Ponder, K. S. Suslick, Angew. Chem. Int. Ed. 2005, 44, 4528-4532; Angew. Chem. 2005, 117, 4604-4608; b) J. R. Askim, M. Mahmoudi, K. S. Suslick, Chem. Soc. Rev. 2013, 42, 8649-8682. 
[7] a) T. L. Nelson, C. O'Sullivan, N. T. Greene, M. S. Maynor, J. J. Lavigne, J. Am. Chem. Soc. 2006, 128, 5640-5641; b) M. S. Maynor, T. L. Nelson, C. O'Sulliva, J. J. Lavigne, Org. Lett. 2007, 9, 3217-3220; c) T. L. Nelson, I. Tran, T. G. Ingallinera, M. S. Maynor, J. J. Lavigne, Analyst 2007, 132, 1024-1030; d) M. Cai, S. L. Daniel, J. J. Lavigne, Chem. Commun. 2013, 49, 6504-6506.

[8] a) S. Nieto, J. M. Dragna, E. V. Anslyn, Chem.-Eur. J. 2010, 16, 227-232; b) J. M. Dragna, G. Pescitelli, L. Tran, V. M. Lynch, E. V. Anslyn, L. Di Bari, J. Am. Chem. Soc. 2012, 134, 4398-4407; c) P. Metola, E. V. Anslyn, T. D. James, S. D. Bull, Chem. Sci. 2012, 3, 156-161; d) Y. T. Zhou, Y. L. Ren, L. Zhang, L. You, Y. F. Yuan, E. V. Anslyn, Tetrahedron 2015, 71, 3515-3521.

[9] B. Lee, R. Scopelliti, K. Severin, Chem. Commun. 2011, 47, 9639-9641.

[10] J. Lim, O. S. Miljanić, Chem. Commun. 2012, 48, 10301-10303.

[11] J. H. Jung, S. J. Lee, J. S. Kim, W. S. Lee, Y. Sakata, T. Kaneda, Org. Lett. 2006, 8, 3009-3012.

[12] P. Q. Leng, F. L. Zhao, B. C. Yin, B. C. Ye, Chem. Commun. 2015, 51, 8712-8714.

[13] a) N. Shao, J. Y. Jin, S. M. Cheung, R. H. Yang, W. H. Chan, T. Mo, Angew. Chem. Int. Ed. 2006, 45, 4944-4948; Angew. Chem. 2006, 118, 5066-5070; b) Y. Shiraishi, K. Adachi, M. Itoh, T. Hirai, Org. Lett. 2009, 11, 3482-3485; c) F. Nourmohammadian, T. Wu, N. R. Branda, Chem. Commun. 2011, 47, $10954-$ 10956; d) Y. Shiraishi, K. Yamamoto, S. Sumiya, T. Hirai, Phys. Chem. Chem. Phys. 2014, 16, 12137-12142. [14] a) M. Irie, Chem. Rev. 2000, 100, 1685-1716; b) H. Tian, S. J. Yang, Chem. Soc. Rev. 2004, 33, 8597.

[15] a) D. Wilson, N. R. Branda, Angew. Chem. Int. Ed. 2012, 51, 5431-5434; Angew. Chem. 2012, 124, 5527-5530; b) M. Kathan, P. Kovaříček, C. Jurissek, A. Senf, A. Dallmann, A. F. Thünemann, S. Hecht, Angew. Chem. Int. Ed. 2016, 55, 13882-13886; Angew. Chem. 2016, 128, 14086-14090.

[16] C. Godoy-Alcantar, A. K. Yatsimirsky, J. M. Lehn, J. Phys. Org. Chem. 2005, 18, 979-985.

[17] Thermally induced rearrangements of some DAEs to benzothiophenes have been observed by Kobatake: a) S. Kobatake, H. Imagawa, H. Nakatani, S. Nakashima, New J. Chem. 2009, 33, 1362-1367; b) D. Kitagawa, S. Kobatake, Chem. Lett. 2011, 40, 93-95; c) H. Shoji, S. Kobatake, Chem. Commun. 2013, 49, 2362-2364.

[18] CCDC 1508362 contains the supplementary crystallographic data for this paper. These data can be obtained free of charge from The Cambridge Crystallographic Data Centre via www.ccdc.cam.ac.uk/data_request/cif.

[19] R. G. Kallen, W. P. Jencks, J. Biol. Chem. 1966, 241, 5864-5878. 


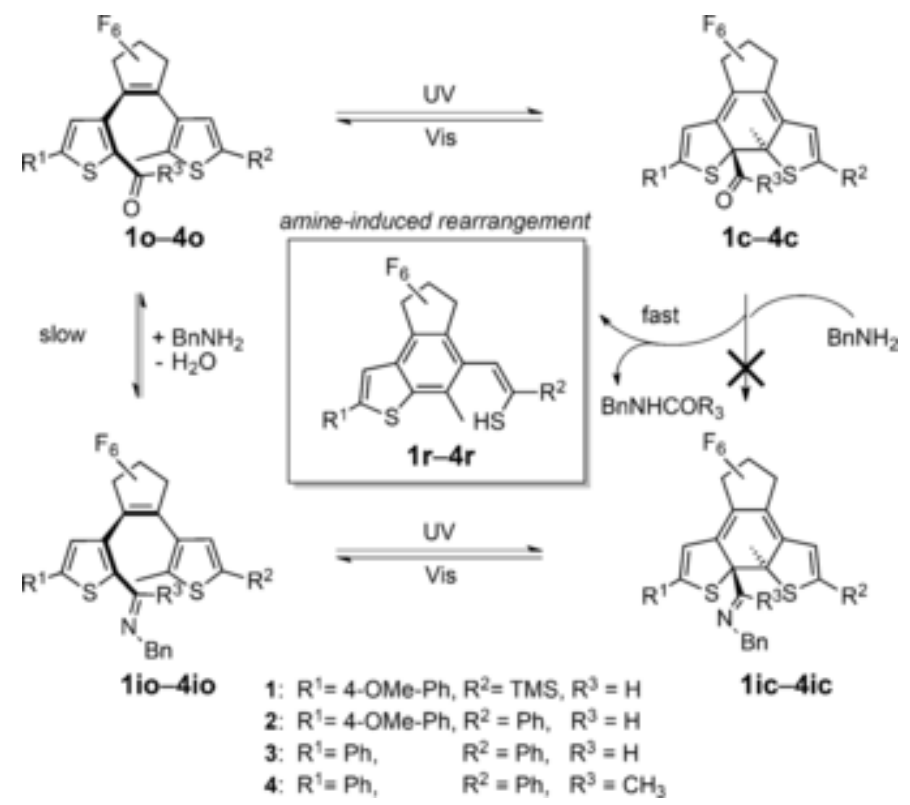

Scheme 1. Light controls the reactivity of open (1o-4o) and closed (1c-4c) diarylethene isomers, carrying aromatic and aliphatic aldehyde/ketone groups, respectively, towards amines resulting in either imines (1i-4i) or benzothiophene rearrangement products $(\mathbf{1 r}-\mathbf{4 r})$. o: open, c: closed, i: imine, r: rearranged. Bn=Benzyl, TMS=trimethylsilyl.
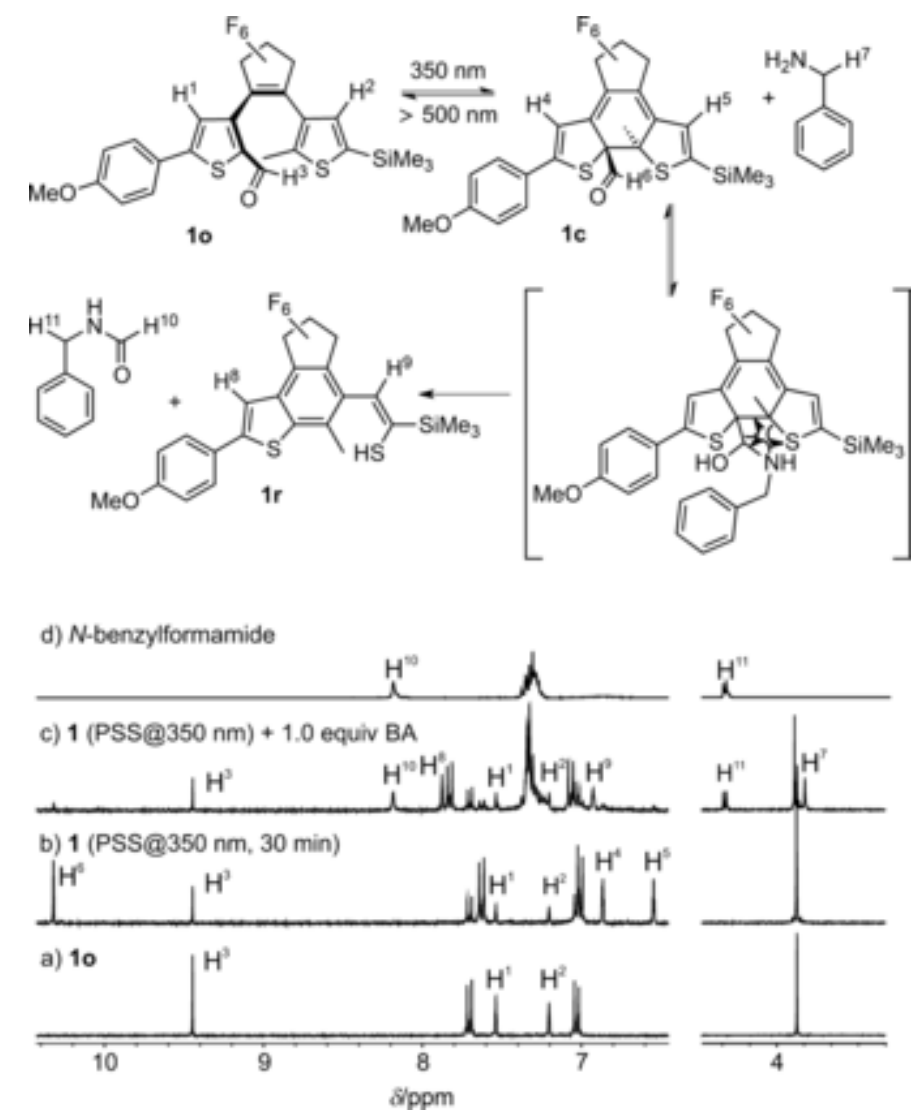

Figure 1. Top: Proposed mechanism for the amine-induced decoloration of aldehyde-substituted DAEs. Bottom: Selected regions of the ${ }^{1} \mathrm{H}$ NMR spectra $\left(\mathrm{CD}_{3} \mathrm{CN}, 298 \mathrm{~K}\right)$ of a) 1o; b) mixture of 10 and 1c corresponding to the PSS after $30 \mathrm{~min}$ irradiation with $350 \mathrm{~nm}$ light; c) mixture of $\mathbf{1 0}$ and $\mathbf{1 c}$ corresponding to the PSS of 10 after $30 \mathrm{~min}$ irradiation with $350 \mathrm{~nm}$ light +1 equiv BA; d) pure $N$-benzylformamide. 

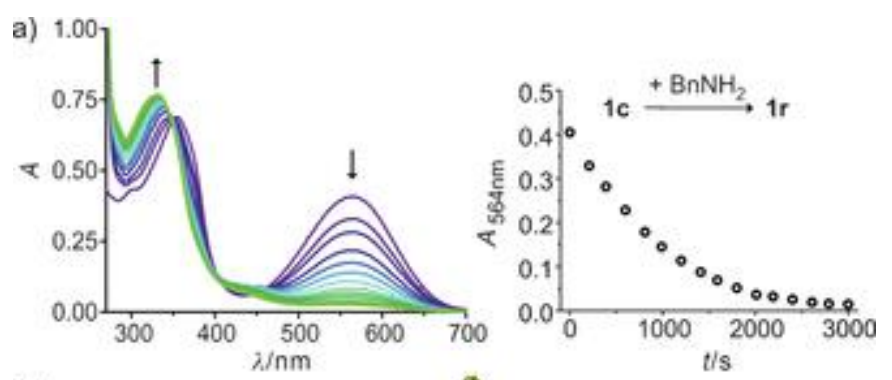

b)

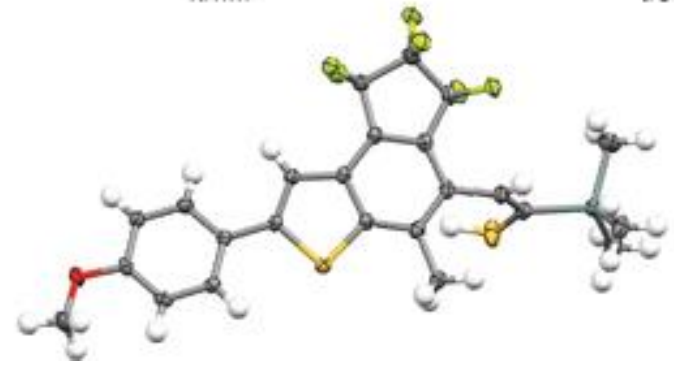

Figure 2. a) Left: Evolution of the UV/Vis absorption spectrum corresponding to the PSS of $1\left(2.4 \times 10^{-5} \mathrm{M}\right)$ after the addition of BA $\left(1.7 \times 10^{-2} \mathrm{M}\right)$. Right: Gradual decay of the band at $564 \mathrm{~nm}$ corresponding to the formation of 1r. b) X-ray crystal structure of 1r; gray $\mathrm{C}$, white $\mathrm{H}$, green $\mathrm{F}$, red $\mathrm{O}$, yellow S. Ellipsoids represent the $50 \%$ probability level. Hydrogen atoms are shown as spheres of arbitrary radii. ${ }^{[18]}$

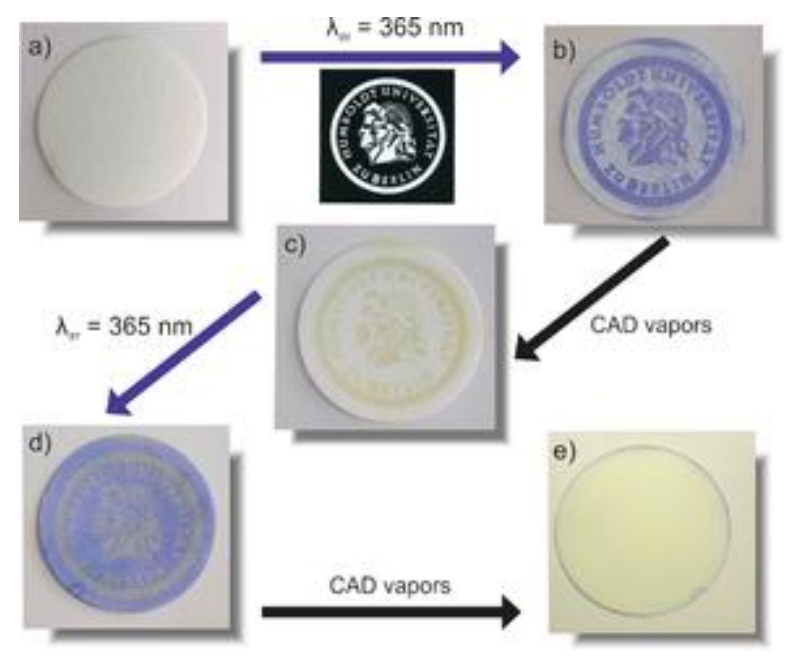

Figure 3. Amine sensing with light-activated filter paper: a) Filter paper coated with a solution of 3o; b) in situ activation by irradiating at $365 \mathrm{~nm}$ for 2 min with a UV lamp after the surface had been covered with a photomask (printed on transparent plastic sheet with a common laser printer); c) color evolution after exposure of the filter paper to CAD vapors for $5 \mathrm{~min}$; d) after removal of the CAD, the non-irradiated areas (residual 3o) can be activated by irradiating at $365 \mathrm{~nm}$ for $2 \mathrm{~min}$, creating the "negative" of picture (b); e) color evolution when the filter paper is exposed again to CAD vapors for $5 \mathrm{~min}$. 
Table 1. First-order rate constants $(\mathbf{k})$ determined for the rearrangement reaction of DAEs $1 \mathbf{c}$ and $3 \mathbf{c}$ with various amines.

\begin{tabular}{|c|c|c|c|}
\hline $\operatorname{Amine}^{[a]}$ & Structure & $\mathbf{k}(\mathbf{1 c}) / \min ^{[\mathrm{b}]}$ & $\mathbf{k}(\mathbf{3 c}) / \min ^{[b]}$ \\
\hline$\overline{\text { SPER }}$ & & 1.67 & 2.66 \\
\hline CAD & & 0.30 & 0.50 \\
\hline DAP & $\mathrm{H}_{2} \mathrm{~N}^{-}$ & 0.49 & 1.58 \\
\hline OA & & 0.12 & 0.13 \\
\hline BA & $\mathrm{H}_{2} \mathrm{~N}^{-}$ & 0.033 & 0.025 \\
\hline MBA & & 0.002 & 0.0012 \\
\hline BMA & $\mathrm{N}^{-}$ & 0.0010 & 0.0007 \\
\hline DMBA & & n.r. & n.r. \\
\hline
\end{tabular}

[a] $1 \times 10^{-2} \mathrm{M}$ in $\mathrm{CH}_{3} \mathrm{CN}$. [b] $2 \times 10^{-5} \mathrm{M}$ in $\mathrm{CH}_{3} \mathrm{CN}$. 